\title{
AC-DC Isolated Matrix Converter Charger: Topology and Modulation
}

Luca Rovere

PEMC

University of Nottingham

Nottingham, UK

luca.rovere@nottingham.ac.uk

Pericle Zanchetta

PEMC,

University of Nottingham,

Nottingham, UK,

Dept. of Electrical,Computer

and Biomedical Engineering

University of Pavia, Pavia, IT

pericle.zanchetta@nottingham.ac.uk,

pericle.zanchetta@unipv.it

\author{
Sabino Pipolo \\ PEMC \\ University of Nottingham \\ Nottingham, UK \\ sabino.pipolo@nottingham.ac.uk
}

\author{
Andrea Formentini \\ PEMC \\ University of Nottingham \\ Nottingham, UK \\ andrea.formentini@nottingham.ac.uk
}

\begin{abstract}
This paper presents the modulation strategy for the $25 \mathrm{~kW}$ AC-DC isolated Matrix Charger three-phase rectifier (MCharger). The proposed topology allows current and voltage regulation for energy storage devices such as EVs batteries. Compared to a standard Dual-Active-Bridge topology, the Matrix converter allows to directly perform an AC-AC energy conversion thus eliminating the rectifying stage. A high frequency (HF) voltage waveform is generated to feed the isolation transformer, which is characterized by an almost negligible leakage inductance, allowing for the reduction of the transformer voltage drops. The rectification of the ac-waveform is achieved at the output stage, consisting of an H-bridge and an LC filter. The suggested topology powered with the proposed modulation is able to provide a wide output voltage range which can vary from $50 \mathrm{~V}$ to $500 \mathrm{~V}$, providing up to a maximum power of $25 \mathrm{~kW}$ allowed for level I chargers.
\end{abstract}

Index Terms-Matrix converter, Modulation strategy, AC-DC, Battery charger, Efficiency

\section{INTRODUCTION}

In the recent years the amount of applications which require dc-power is increasing significantly.

Based on the application type, the ac-dc converter can be divided according to the possibility to provide or not the galvanic isolation between grid and load [1], [2]. However, to ensure the safety of operation and allow for voltage levels adaptation, the distribution system operator (DSO) is more often requiring the grid-connected converter operator to provide for an isolation stage. The growth of Electric Vehicles (EVs) demand is driving the research towards more innovative solutions which increases the efficiency, improve the power density and reduces the cost per $\mathrm{kW}$ installed of modern power electronics converters [3]-[5].
The isolated ac-dc converter topology can be grouped in two stages and single-stage conversion systems. The main disadvantage of the double stage system is the input rectifier which does not allow for the input mains current to be sinusoidal [6] and involves the use of capacitors also on the primary side of the converter. To overcome this problem, the input rectifier and the primary side Full-bridge can be replaced by a $3 \times 2$ direct matrix converter (DMC) [7], [8].

DMC also allows having a bidirectional power flow by converting the input ac-voltage into a high-frequency acvoltage supplied to a high-frequency isolation transformer. The secondary voltage is then rectified to the desired dc-output voltage [9]. In a DMC buck-rectifier with a diode bridge and an additional output inductor is also investigated in [10] where the comprehensive analysis of different switching patterns for the DMC is presented. Here the authors analyse the switching pattern in order to achieve zero voltage switching (ZVS) for a sufficiently high output power demand. In [11] a zero voltage and zero current modulation scheme has been proposed for the DAB buck-boost power factor corrector (PFC) DMC rectifier converter where an isolation transformer with sufficiently large leakage inductance is adopted.

In this paper, an ac-dc isolated single-stage rectifier with DMC on grid side is presented. On the contrary of the previous topologies presented in the literature, the proposed one achieves the power transfer not as a conventional DAB converter, exploiting the HF transformer leakage inductance $L_{l}$, but using the output inductor $L_{o}$. For this reason, $L_{l}$ is kept as low as possible and the output stage $\mathrm{H}$-bridge is used exclusively to rectify the DMC ac voltage waveform. The proposed AC-DC converter is able to operate from a $230 V_{r m s}$ grid and deliver a dc-output voltage which ranges from 50 


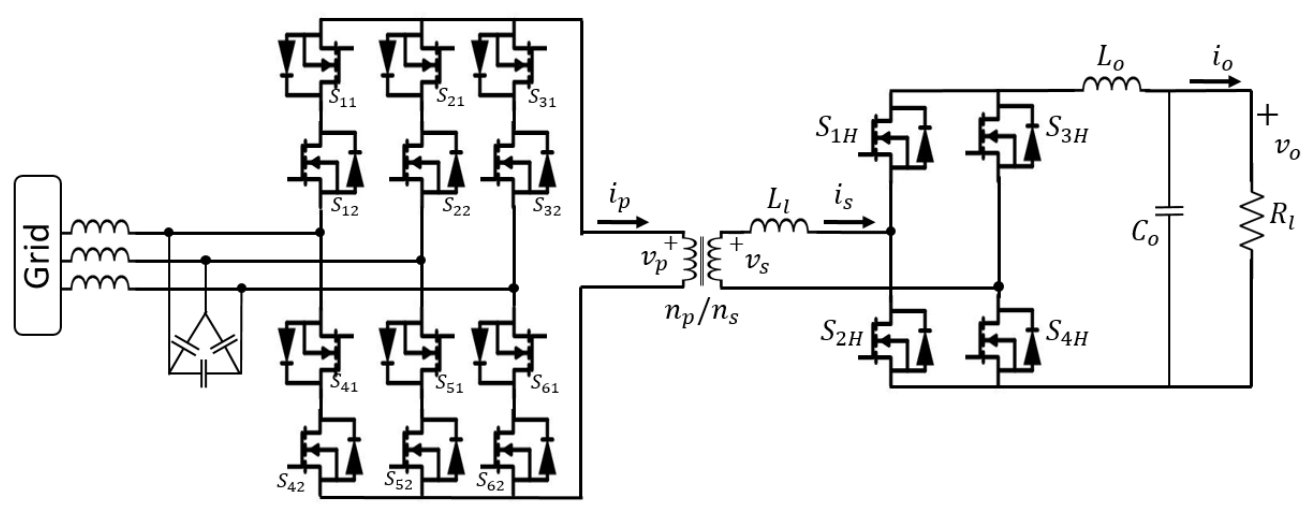

Fig. 1. Isolated single-stage three phase rectifier. The first stage consists in an AC-AC conversion, which is achieved by a $3 \times 2$ DMC able to generate any desired AC voltage waveform $v_{p}$. The transformer provide galvanic isolation and voltage requirement matching by appropriate turn ratio selection. The output stage consists of a H-bridge which allows for bidirectional power flow and a LC filter

to $500 \mathrm{~V}$. In Sections 2 and 3 the topology and the specific modulation developed for its operation are presented.

\section{MCHARGER TOPOLOGY}

Fig. 1 shows the proposed AC-DC MCharger topology. The DMC works as a voltage source converter which supplies the AC voltage $v_{p}$ to the primary of the HF isolation transformer making sure to maintain the volt-second balance. The HF transformer can be modelled exclusively with its leakage inductance $L_{l}$ and its transformer ratio $n=n_{p} / n_{s}$. The resistance of the transformer windings is neglected since the focus is not on the losses determination, while even though the magnetising inductance causes reactive power in the $\mathrm{HF} \mathrm{AC}$ part of the charger, its impact on $\mathrm{AC}$ the current waveforms $i_{p}$ and $i_{s}$ is neglected. Due to the series connection of the output filter inductor and the transformer, in order to allow for a fast change of polarity of the ac transformer current its leakage inductance $L_{1}$ must be sufficiently small. In this way the voltage waveform $v_{p}$ generated by the DMC is directly applied to the output load after rectification by the H-bridge stage and filtering. The low leakage inductance allows for fast polarity inversion of the $\mathrm{AC}$ transformer current during the inversion of the $v_{p} \mathrm{AC}$ voltage. The transformer inductance and the output filter one, $L_{o}$, are series connected, therefore to avoid any over-voltage due to the breaking of an inductive current the $\mathrm{H}$-bridge serves as electrical node for the transformer current to invert its polarity. The specific modulation which allows for the generation of the desired output voltage and manages the $\mathrm{AC}$ transformer current polarity is described in detail in the following section.

\section{MODUlation}

The modulation of the single stage AC-DC charger is here described, the common drain MOSFET of the DMC topology are simplified with ideal switches as shown in Fig 2.

The final implementation of the modulation requires the well known 4-STEP switching procedure in order to generate the 12 gate logic signals starting from the 6 ones generated by the modulation. Furthermore, additional simplification can be

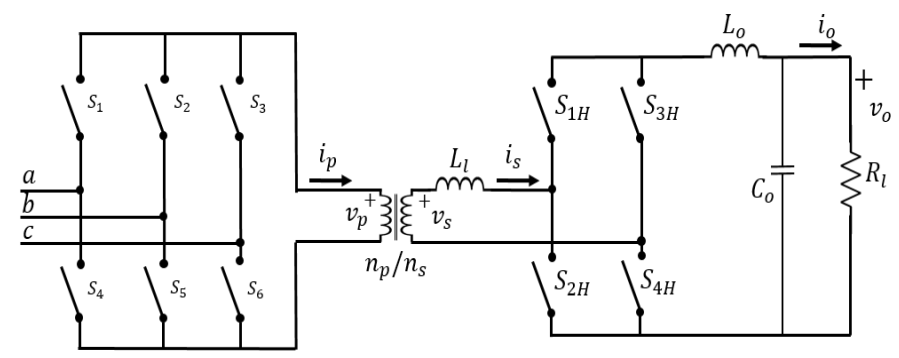

Fig. 2. Simplified schematic of the MCharger where the DMC MOSFETs are replaced with ideal switches $S_{i}$.

achieved by omitting the input LC filter (which does not have any impact on the modulation derivation). The modulation strategy is here derived for the portion of the grid electrical period where the phase voltages are in the following order:

$$
v_{a}>v_{b}>v_{c}
$$

Extension to the other grid angles comes automatically by correctly ordering the input phase voltages in decreasing order according to their amplitude [12]. The first step for the modulation calculation is the determination of the voltage vectors duties of the $3 \times 2$ DMC. Considering that the output voltage seen by the load corresponds to the rectified voltage generated by the DMC at the transformer input, the following condition can be stated:

$$
v_{o}^{*}=\frac{1}{T_{s}} \int_{0}^{T_{s}} v_{p}(t) d t
$$

where $v_{o}^{*}$ is the output reference voltage and $T_{s}$ is the controller sample time which corresponds to half the period $T_{c}$ of the DMC ac output voltage waveform. Equation (2) can be written considering exclusively the three active vectors which at each sample time are used to synthesize the output reference voltage obtaining:

$$
v_{o}^{*}=d_{1}\left(v_{a}-v_{b}\right)+d_{2}\left(v_{b}-v_{c}\right)+d_{3}\left(v_{c}-v_{a}\right)
$$


In order to achieve unitary power factor at the 3 phase DMC input and in order to prevent short circuit on the input side and ensure uninterrupted load current flow the duty cycles must satisfy also the following relationships:

$$
\left\{\begin{array}{l}
i_{a}=d_{1} i_{p}+d_{3} i_{p} \\
i_{b}=d_{2} i_{p}-d_{1} i_{p}
\end{array}\right.
$$

Finally, two additional constraints have to be considered when resolving the analytical problem to find the duty cycles expressions:

$$
\left\{\begin{array}{l}
v_{a}+v_{b}+v_{c}=0 \\
d_{0}+d_{1}+d_{2}+d_{3}=1
\end{array}\right.
$$

Equations (5) represent the symmetry of the grid voltages and the duty cycles relationship. The determination of the modulation strategy for the matrix converter consists in the problem of determining, in each sample time, the duty cycles that satisfy equations (3), (4) and (5), i.e. the input-output voltage and current relationships.

The duty cycles can now be calculated rearranging (3), (4) and (5) obtaining the final duties' expression

$$
\left\{\begin{array}{l}
d_{1}=\frac{-\left(v_{o}^{*} v_{a}-d_{3} v_{a}^{2}-d_{3} v_{b}^{2}+d_{3} v_{a} v_{c}+d_{3} v_{b} v_{c}\right)}{\left(-v_{a}^{2}+v_{c} v_{a}-v_{b}^{2}+v_{c} v_{b}\right)} \\
d_{2}=\frac{-\left(v_{o}^{*} v_{a}+v_{o}^{*} v_{b}-d_{3} v_{a}^{2}-d_{3} v_{b}^{2}+d_{3} v_{a} v_{c}+d_{3} v_{b} v_{c}\right)}{\left(-v_{a}^{2}+v_{c} v_{a}-v_{b}^{2}+v_{c} v_{b}\right)} \\
d_{3}=\frac{-\left(2 v_{o}^{*} v_{a}+v_{o}^{*} v_{b}-2 d_{3} v_{a}^{2}-2 d_{3} v_{b}^{2}+2 d_{3} v_{a} v_{c}+2 d_{3} v_{b} v_{c}\right)}{\left(-v_{a}^{2}+v_{c} v_{a}-v_{b}^{2}+v_{c} v_{b}\right)} \\
d_{0}=1-d_{1}-d_{2}-d_{3}
\end{array}\right.
$$

It can be noticed that (6) presents $\infty^{1}$ solutions dependent from $d_{0}$. The DMC output voltage vectors according to the input phase voltages ordering are shown in Table I. the voltage vector is shown as a row vector containing the 6 DMC bidirectional switch logic state, where 1 stands for conducting and 0 for open. Referring to Fig. 2, the $3 \times 2$ DMC voltage vector shown in Table I is

$$
v_{i}=\left[\begin{array}{llllll}
S_{1} & S_{2} & S_{3} & S_{4} & S_{5} & S_{6}
\end{array}\right]
$$

where $i=0,1,2,3$. Therefore, the instantaneous output voltage of the ideal $3 \times 2 \mathrm{DMC}$ can be calculated as

$$
v_{p}=\left[\begin{array}{lll}
v_{a} & v_{b} & v_{c}
\end{array}\right]\left[\begin{array}{l}
S_{1}-S_{4} \\
S_{2}-S_{5} \\
S_{3}-S_{6}
\end{array}\right]
$$

An additional degree of freedom when defining the $3 \times 2$ DMC modulation strategy is given by the possibility to arrange the voltage vectors in an arbitrary sequence. The use of different switching patterns for the same duty cycles leads to a different behaviour in terms of number of switch commutations and ripple of input and output quantities. In this work, in order to reduce the switching losses of the DMC AC-AC stage,

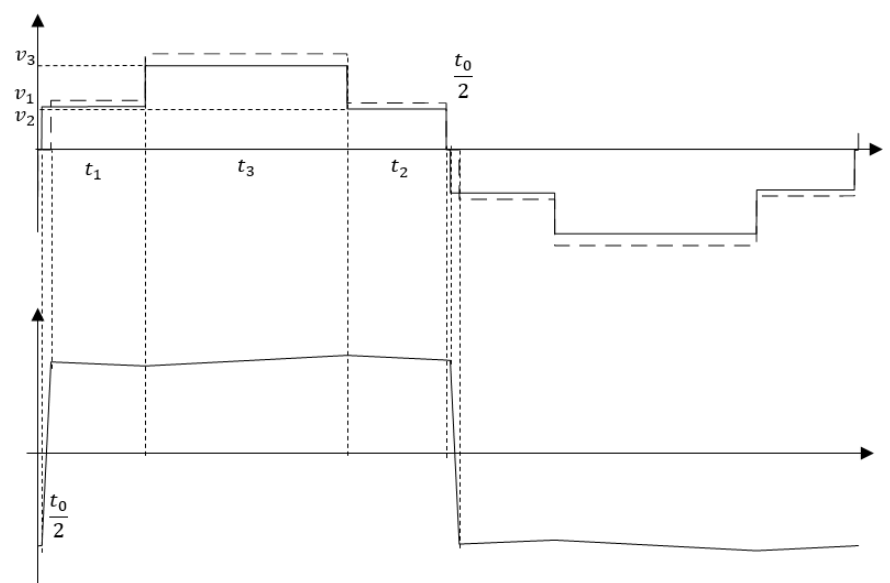

Fig. 3. Steady state voltage waveforms for the voltage vector sequence $v_{0}, v_{1}, v_{3}, v_{2}, v_{0}$. Top: $v_{p}$ solid line, $v_{s}$ dashed line. Bottom: ac transformer current waveform.

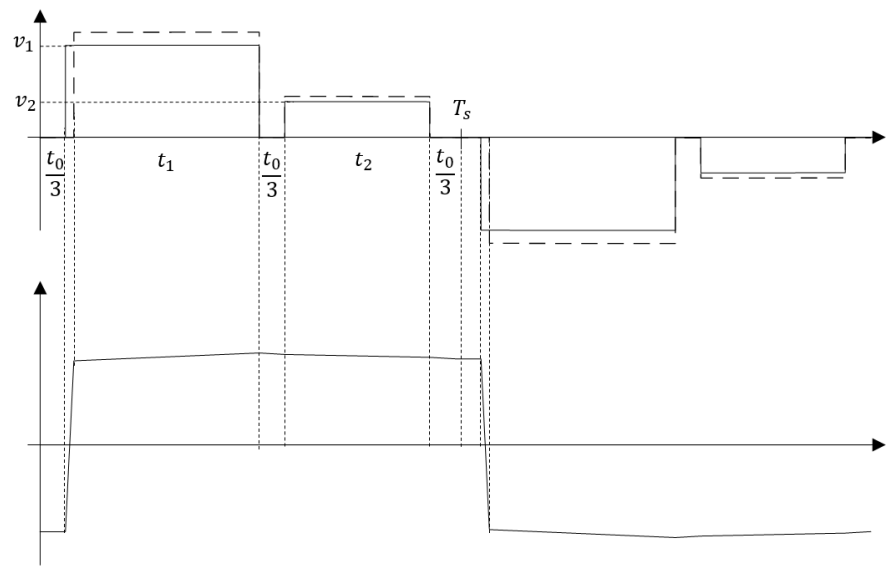

Fig. 4. Steady state voltage waveforms for the voltage vector sequence $v_{0}, v_{1}, v_{0}, v_{2}, v_{0}$. Top: $v_{p}$ solid line, $v_{s}$ dashed line. Bottom: ac transformer current waveform.

an additional constraint is introduced regarding the voltage vectors order of application. Only one device is allowed to switch from on voltage vector transition to the following one. Furthermore, the first non-zero vector, which is applied after the change of voltage polarity, must be at least the active vector with the medium value among the three active voltage vectors. This is to guarantee a fast transformer current inversion and consequently reduce the time for which the input voltage $v_{p}$ is not transferred to the output. The ideal DMC six gate signals corresponding to the voltage vectors $v_{0}, v_{1}, v_{2}$ and $v_{3}$ for each combination of maximum and minimum input phase voltage are reported in Table I for completeness. From the previous stated switching patter constraint and from (6) the duty cycles can be calculated with the following approach: firstly $d_{3}$ is calculated supposing the available degree of freedom $d_{0}$ equal to zero. If the resulting $d_{3}$ is greater than zero, then in order to synthesise the output reference voltage $v_{o}^{*}$ all the three active voltage vectors are necessary. Consequently, according to the 
TABLE I

SWITCHING PATTERN FOR THE DMC

\begin{tabular}{|c|c|c|c|c|c|}
\hline$V_{\text {phase }}^{\max }$ & $V_{\text {phase }}^{\min }$ & $v_{0}$ & $v_{1}$ & $v_{2}$ & $v_{3}$ \\
\hline$V_{a}$ & $V_{c}$ & {$\left[\begin{array}{llllll}0 & 1 & 0 & 0 & 1 & 0\end{array}\right]$} & {$\left[\begin{array}{llllll}0 & 1 & 0 & 0 & 0 & 1\end{array}\right]$} & {$\left[\begin{array}{llllll}1 & 0 & 0 & 0 & 1 & 0\end{array}\right]$} & {$\left[\begin{array}{llllll}1 & 0 & 0 & 0 & 0 & 1\end{array}\right]$} \\
\hline$V_{a}$ & $V_{b}$ & {$\left[\begin{array}{llllll}0 & 0 & 1 & 0 & 0 & 1\end{array}\right]$} & {$\left[\begin{array}{llllll}0 & 0 & 1 & 0 & 1 & 0\end{array}\right]$} & {$\left[\begin{array}{llllll}1 & 0 & 0 & 0 & 0 & 1\end{array}\right]$} & {$\left[\begin{array}{llllll}1 & 0 & 0 & 0 & 1 & 0\end{array}\right]$} \\
\hline$V_{b}$ & $V_{c}$ & {$\left[\begin{array}{llllll}1 & 0 & 0 & 1 & 0 & 0\end{array}\right]$} & {$\left[\begin{array}{llllll}1 & 0 & 0 & 0 & 0 & 1\end{array}\right]$} & {$\left[\begin{array}{llllll}0 & 1 & 0 & 1 & 0 & 0\end{array}\right]$} & {$\left[\begin{array}{llllll}0 & 1 & 0 & 0 & 0 & 1\end{array}\right]$} \\
\hline$V_{b}$ & $V_{a}$ & {$\left[\begin{array}{llllll}0 & 0 & 1 & 0 & 0 & 1\end{array}\right]$} & {$\left[\begin{array}{llllll}0 & 0 & 1 & 1 & 0 & 0\end{array}\right]$} & {$\left[\begin{array}{llllll}0 & 1 & 0 & 0 & 0 & 1\end{array}\right]$} & {$\left[\begin{array}{llllll}0 & 1 & 0 & 1 & 0 & 0\end{array}\right]$} \\
\hline$V_{c}$ & $V_{a}$ & {$\left[\begin{array}{llllll}0 & 1 & 0 & 0 & 1 & 0\end{array}\right]$} & {$\left[\begin{array}{llllll}0 & 1 & 0 & 1 & 0 & 0\end{array}\right]$} & {$\left[\begin{array}{llllll}0 & 0 & 1 & 0 & 1 & 0\end{array}\right]$} & {$\left[\begin{array}{llllll}0 & 0 & 1 & 1 & 0 & 0\end{array}\right]$} \\
\hline$V_{c}$ & $V_{b}$ & {$\left[\begin{array}{llllll}1 & 0 & 0 & 1 & 0 & 0\end{array}\right]$} & {$\left[\begin{array}{llllll}1 & 0 & 0 & 0 & 1 & 0\end{array}\right]$} & {$\left[\begin{array}{llllll}0 & 0 & 1 & 1 & 0 & 0\end{array}\right]$} & {$\left[\begin{array}{llllll}0 & 0 & 1 & 0 & 1 & 0\end{array}\right]$} \\
\hline
\end{tabular}

switching constraint introduces, the selected voltage vectors sequence will be $v_{0}, v_{1}, v_{3}, v_{2}, v_{0}$ for the considered sample time. Otherwise if $d_{3}$ is smaller than zero the voltage vector $v_{3}$ is not necessary, i.e. the output reference voltage is smaller than the medium input line voltage, and the selected voltage vectors sequence will be $v_{0}, v_{1}, v_{0}, v_{2}, v_{0}$. The corresponding voltage waveforms for the two cases are shown in Fig. 3 and Fig. 4.

\section{A. H-Bridge Modulation}

At the beginning of each sample time $T_{s}$ the H-bridge is commuted either in direct connection, i.e. where $S_{1 H}$ and $S_{4 H}$ are on, or inverse connection, $S_{2 H}$ and $S_{3 H}$ are on. After the zero voltage vector is applied an active voltage vector of opposite polarity of the ac circulating current is applied. During the time of inversion of the ac current, i.e. from the time instant $t_{1}$ to $t_{3}$ in Fig. 5, the H-bridge acts as a shortcircuit decoupling the dynamics of the two inductive currents $i_{s}$ and $i_{o}$. In fact, during this time the voltage at the input of the H-bridge is zero, it rises to $v_{p} / n$ only after $i_{s}$ has completely reversed, i.e. until $i_{s}$ is not in module equal to the output current $i_{o}$. The detailed current paths can be observed in Fig. 6 where a transition to negative $i_{s}$ is shown.

\section{Simulation Results}

The simulation are performed with the aim to highlight the ability of the converter to provide a maximum rated power of $25 \mathrm{~kW}$ with a wide range of the output voltage from a minimum of $50 \mathrm{~V}$ to a maximum of $500 \mathrm{~V}$. The transformer turn ratio $n$ has been selected to be 0.875 and its leakage inductance $L_{l}$ is $1 \mu \mathrm{H}$. The output filter stage has been selected as a trade off between components volumes and output quantities ripple resulting in $L_{o}$ of $180 \mu \mathrm{H}$ and $C_{o}$ of $50 \mu \mathrm{H}$.

In Fig.7 the operation acquired in a grid period is shown, where it can be appreciated the primary transformer voltage $v_{p}$ (pink), the output current (cyan) and the input line voltages. Fig. 8 show a magnification where is visible how the $v_{p}$ is built when the line voltages are as $V_{a b}>V_{b c}>V_{c a}$.

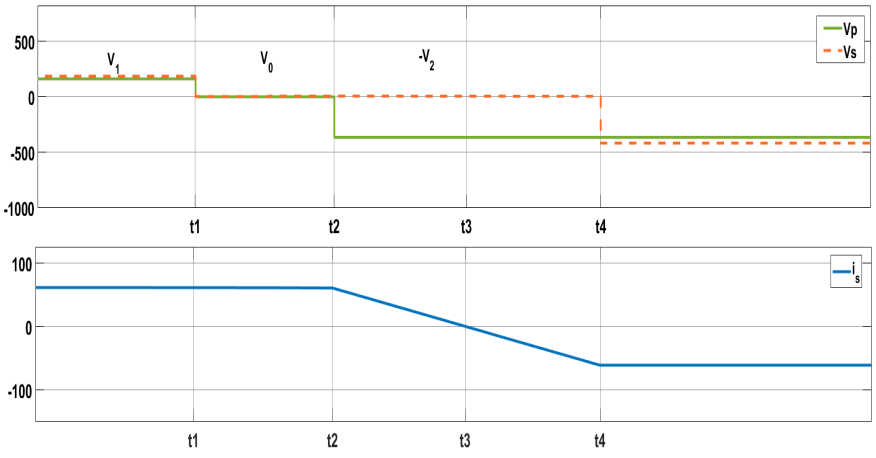

Fig. 5. Transformer current polarity inversion. (1) Top: ac-primary voltage $v_{p}$ (green solid line),ac-secondary voltage $v_{s}$ (red dashed line). Bottom: acsecondary current waveform $i_{s}$ (blue line).

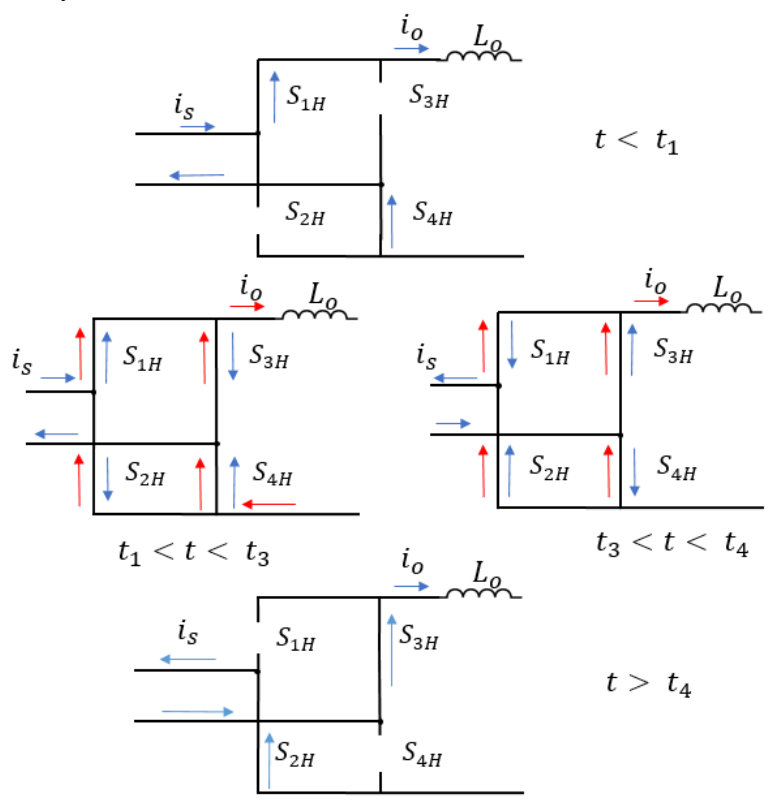

Fig. 6. $i_{o}$ and $i_{s}$ currents paths through the H-bridge during the polarity change. 


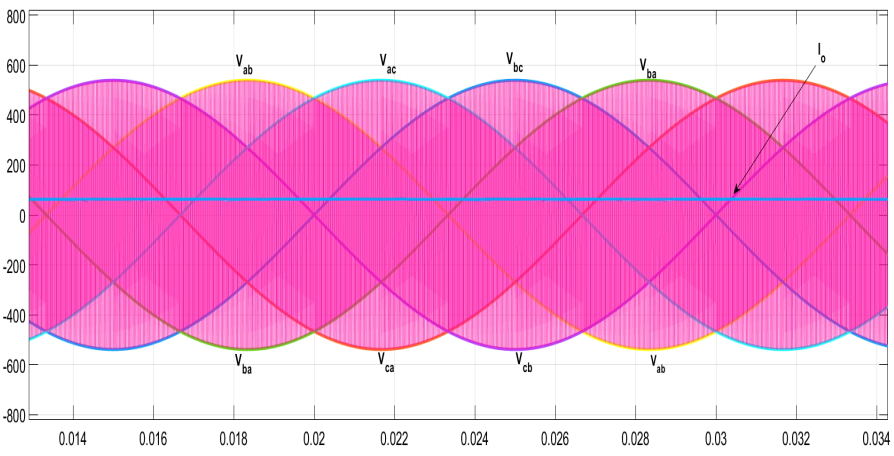

Fig. 7. Input line voltages, ac-primary transformer voltage (pink), output current (cyan).

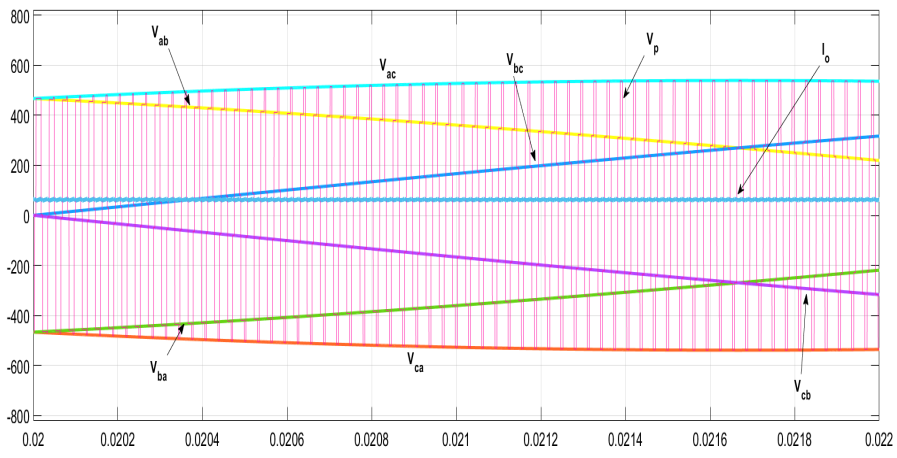

Fig. 8. Input line voltages, primary transformer voltage (pink), output current (cyan).

Fig. 9 and 10 show the simulation results of the steadystate voltage and current waveforms for a requested output voltage of $250 \mathrm{~V}$ and $450 \mathrm{~V}$ respectively. In particular, Fig. 10 represents the operative point where the duty cycle associated to the maximum phase voltage $d_{3}$ is greater than zero, so the voltage vectors sequence is $v_{0}, v_{1}, v_{3}, v_{2}, v_{0}$. On the contrary, Fig. 9 shows the operative point where the output voltage can be synthesised by using only the medium and minimum voltages.

In Fig.11, a step response on output voltage is shown. More in detail, at the time T1 and T2, two steps to $250 \mathrm{~V}$ and 500 $\mathrm{V}$ are required respectively. By imposing a constant ohmic load, it can be appreciated that the converter is able to provide the maximum power at maximum output voltage reaching 50 Amps output current and a $25 \mathrm{~kW}$ output power.

\section{CONCLUSIONS}

In this paper, an isolated AC-DC single-stage topology is presented. The proposed structure is powered with the Direct Matrix converter on the grid side which allows to provide directly a high frequency waveform on the primary of of an isolation transformer. The output stage is composed with an $\mathrm{H}-$ bridge converter plus an LC filter. Despite the previous topologies presented in the literature, the proposed one achieves the power transfer not as a conventional DAB converter, ex-

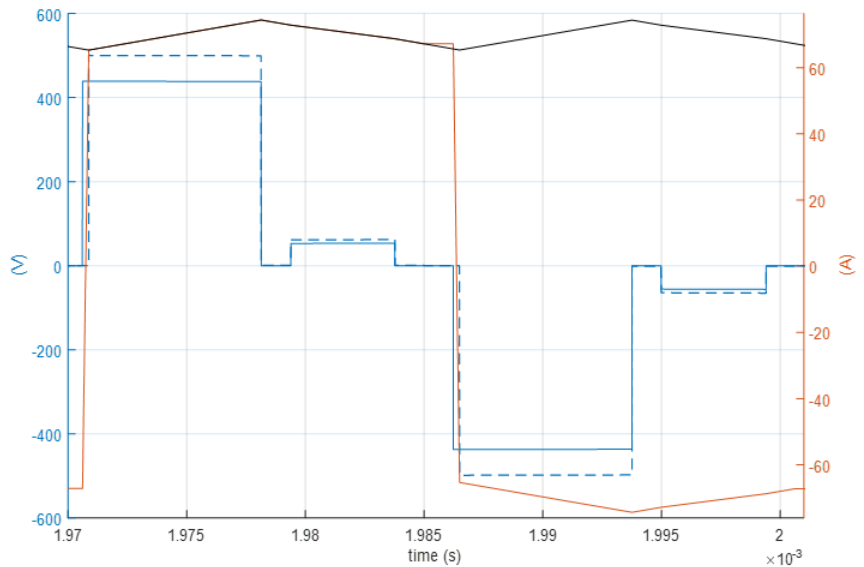

Fig. 9. Steady state waveforms with $v_{o}$ set to $250 \mathrm{~V}$. $v_{p}$ solid blue line, $v_{s}$ dashed blue line, $i_{s}$ solid red line, $i_{o}$ solid black line.

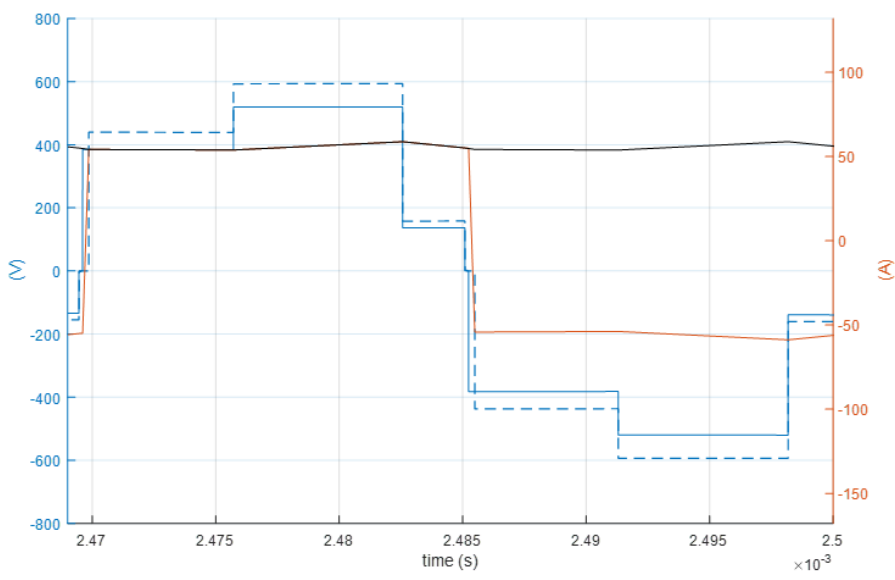

Fig. 10. Steady state waveforms with $v_{o}$ set to $450 \mathrm{~V} . v_{p}$ solid blue line, $v_{s}$ dashed blue line, $i_{s}$ solid red line, $i_{o}$ solid black line.
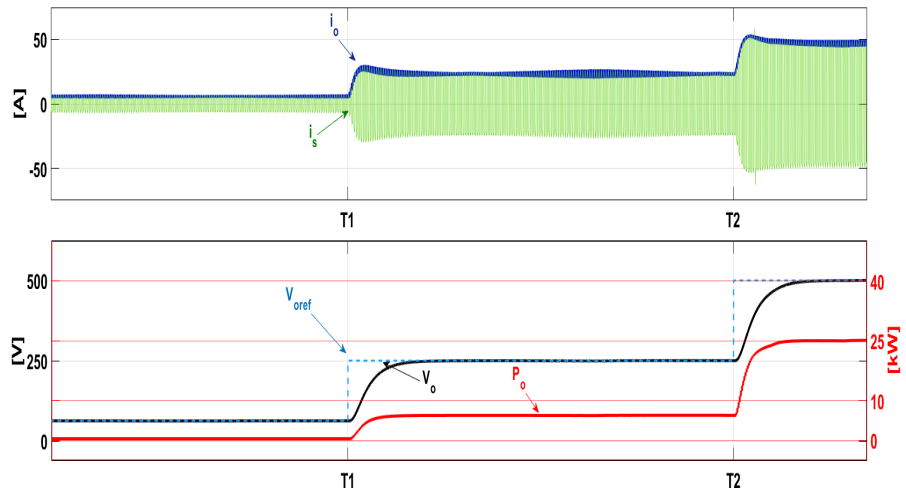

Fig. 11. Step response waveforms with $V_{o}$ set to $50 \mathrm{~V}, 250 \mathrm{~V}$ and $500 \mathrm{~V}$. Top: ac-secondary transformer voltage $v_{s}$, output current $i_{o}$. Bottom: output voltage reference $V_{\text {oref }}$, output voltage $V_{o}$, output power $P_{o}$.

ploiting the HF transformer leakage inductance, but using the output inductor. For this reason, the HF transformer leakage inductance is designed as low as possible allowing for the 
reduction of the transformer voltage drops. The output stage is used exclusively to rectify the DMC ac voltage waveform. The dedicated modulation strategy developed for its operation have been presented and the promising simulation results have shown that the proposed AC-DC converter is able to operate at $230 V_{r m s}$ grid delivering a dc-output voltage which ranges from $50 \mathrm{~V}$ to $500 \mathrm{~V}$. Since the converter can provide up to a maximum power of $25 \mathrm{~kW}$, it can be included in the level I chargers.

\section{REFERENCES}

[1] M. Mengoni, L. Zarri, A. Tani, G. Rizzoli, G. Serra, and D. Casadei, "Modulation strategies for three-phase ac-dc matrix converters: A comparison," in 2016 IEEE Energy Conversion Congress and Exposition (ECCE). IEEE, 2016, pp. 1-7.

[2] L. Schrittwieser, P. Cortés, L. Fässler, D. Bortis, and J. W. Kolar, "Modulation and control of a three-phase phase-modular isolated matrixtype pfc rectifier," IEEE Transactions on Power Electronics, vol. 33, no. 6, pp. 4703-4715, 2017.

[3] T. Dragičević, X. Lu, J. C. Vasquez, and J. M. Guerrero, "Dc microgrids-part ii: A review of power architectures, applications, and standardization issues," IEEE Transactions on Power Electronics, vol. 31, no. 5, pp. 3528-3549, May 2016.

[4] S. S. Williamson, A. K. Rathore, and F. Musavi, "Industrial electronics for electric transportation: Current state-of-the-art and future challenges," IEEE Transactions on Industrial Electronics, vol. 62, no. 5 , pp. 3021-3032, May 2015.

[5] A. K. Singh, E. Jeyasankar, P. Das, and S. K. Panda, "A single-stage matrix-based isolated three-phase ac-dc converter with novel current commutation," IEEE Transactions on Transportation Electrification, vol. 3, no. 4, pp. 814-830, 2016.

[6] J. Everts, F. Krismer, J. Van den Keybus, J. Driesen, and J. W. Kolar, "Comparative evaluation of soft-switching, bidirectional, isolated ac/dc converter topologies," in 2012 Twenty-Seventh Annual IEEE Applied Power Electronics Conference and Exposition (APEC), Feb 2012, pp. 1067-1074.

[7] N. D. Weise, K. K. Mohapatra, and N. Mohan, "Universal utility interface for plug-in hybrid electric vehicles with vehicle-to-grid functionality," in IEEE PES General Meeting, July 2010, pp. 1-8.

[8] D. Varajão, R. E. Araújo, L. M. Miranda, and J. A. P. Lopes, "Modulation strategy for a single-stage bidirectional and isolated ac-dc matrix converter for energy storage systems," IEEE Transactions on Industrial Electronics, vol. 65, no. 4, pp. 3458-3468, April 2018.

[9] R. García-Gil, J. M. Espi, E. J. Dede, and E. Sanchis-Kilders, "A bidirectional and isolated three-phase rectifier with soft-switching operation," IEEE Transactions on Industrial Electronics, vol. 52, no. 3, pp. 765-773, 2005.

[10] J. Afsharian, D. Xu, B. Wu, B. Gong, and Z. Yang, "The optimal pwm modulation and commutation scheme for a three-phase isolated buck matrix-type rectifier," IEEE Transactions on Power Electronics, vol. 33 , no. 1, pp. 110-124, Jan 2018.

[11] L. Schrittwieser, M. Leibl, and J. W. Kolar, "99three-phase matrix-type dab buck-boost pfc rectifier," IEEE Transactions on Power Electronics, vol. 35, no. 1, pp. 138-157, Jan 2020.

[12] S. Pipolo, A. Formentini, A. Trentin, P. Zanchetta, M. Calvini, and M. Venturini, "A new modulation approach for matrix converter," in 2019 10th International Conference on Power Electronics and ECCE Asia (ICPE 2019-ECCE Asia). IEEE, 2019, pp. 1021-1027. 